\title{
Bridge Burst Characteristics of Aluminum and Copper Thin-Film Bridges in Electrical Initiation Devices
}

\author{
Kyoungjin Kim ${ }^{1}$, Kyu-Hyoung $\mathrm{Kim}^{2}$, and Seung-gyo Jang,* \\ ${ }^{1}$ Department of Mechanical System Engineering, Kumoh National Institute of Technology, Gumi 39177, Republic of Korea \\ ${ }^{2}$ Defense R\&D Center, Hanwha Corporation, Daejeon 34060, Republic of Korea \\ ${ }^{3}$ Agency for Defense Development, Daejeon 34186, Republic of Korea
}

\begin{abstract}
The optimal design of electrical initiation devices for explosive charges and other high energy materials critically depends on the bridge burst or the electrical explosion of the thin-film metallic bridges. It is therefore quite important to select the right material with suitable explosion properties for the metallic bridge film. However, so far, no dedicated studies, experimental or theoretical, have been carried out to quantify those explosion properties for thin-filmed bridges of various metals. This study employed numerical modeling of the joule heating and bridge plasma expansion by intense electrical energy deposition in aluminum and copper bridges to evaluate and compare the explosion properties such as bridge burst time, bridge burst current density, and threshold charging voltage for the two metallic bridge materials. The prediction model of bridge burst also considered a pulsed electrical input from an RLC initiation circuit as well as plasma property calculations of the aluminum and copper plasma vapor for the state equation of the ionized vapor and the plasma electrical conductivity. The numerical predictions were in excellent agreement with the corresponding measured data of bridge bursts in a series of exploding foil initiator firing tests. The copper bridge was found to possess better explosion properties than the aluminum bridge, showing significantly higher bridge burst current density and threshold charging voltage. These findings confirmed that the copper bridge may provide higher safety and reliability in electrical initiation devices. The theoretical model and results in the present study could be useful for selecting bridge materials and designing electrical initiators or other metallic bridge explosion applications.
\end{abstract}

(Received December 4, 2017; Accepted January 10, 2018)

Keywords: metallic bridge, bridge burst, aluminum, copper, plasma, electrical initiator

\section{INTRODUCTION}

Electrical initiation devices for the detonation or initiation of high energy materials such as explosives and propellants play crucial roles in various military and civilian applications. They may take several forms, including exploding bridgewire (EBW), exploding bridge foil (EBF), and exploding foil initiator (EFI). EBW or EBF use a pulsed form of electrical energy from an initiation circuit and generate a violent expansion and shock wave of high temperature and high pressure plasma by vaporizing a small sized

*Corresponding Author: Seung-gyo Jang [Tel: +82-42-821-4580, E-mail: jsg4580@add.re.kr] Copyright (c) The Korean Institute of Metals and Materials metal wire or foil in a very short duration of energy release time. EFI is structurally similar to EBF but it differs from $\mathrm{EBW}$ or $\mathrm{EBF}$ by placing a thin plastic film over the thin-film bridge and creating high speed impact of small plastic flyer for the explosive charge detonation [1,2].

The explosive or propellant initiators in weapon systems or spacecrafts must maintain extreme levels of reliability and safety, and it is therefore important to select the right materials for the various components in the initiators. Among them, the selection of the metallic bridge materials is particularly crucial in addition to its shape and dimensions (size and thickness), because the detonation performance and reliability greatly depends on the bridge vaporization and plasma expansion. In such devices, the bridge 
film first experiences intense joule heating as the pulsed energy passes by and, then, it undergoes fast phase changes to vapor and plasma in a very short time duration of a few microseconds or even less than a microsecond. This subsequently leads to the rapid and violent expansion of the bridge material. This physical phenomenon, the electrical explosion of the thin metallic foil, is usually referred as the bridge burst. Electrical exploding of small sized metallic conductors can also be used in novel applications in other technical fields, including nanoparticle production, the precision joining of metal and ceramic specimens, and fast-acting opening switches [3].

The thin-film bridge is typically made of aluminum or copper and, though less frequently, gold and silver have been tried as bridge materials, as these metals possess high electrical conductivity and good explosion properties [4]. The selection of bridge materials is usually conducted through a series of initiator tests and experiments, however, the rapid changes of bridge burst in small scale systems make any measurements quite difficult. Therefore, this study aims to investigate the burst characteristics on metallic bridges of aluminum and copper using theoretical approach and compare the physical details of the explosion properties in those metallic bridges.

\section{THEORETICAL APPROACH ON BRIDGE BURST}

Since the current and advanced forms of electrical initiators mainly employ the metallic bridge of thinfilm type, this study focuses on aluminum and copper bridge bursts in an EFI detonation system. As shown in the EFI schematic of Fig. 1, the metallic bridge of square shape is connected to an initiation circuit, which provides a pulsed electrical energy from a capacitor when it is switched on. In this form of explosive detonation system, a thin plastic film (usually polyimide) is placed over the bridge and a small piece of plastic flyer shears out of the plastic film when the bridge burst occurs and the expanding pressure of the bridge plasma builds up. There exists

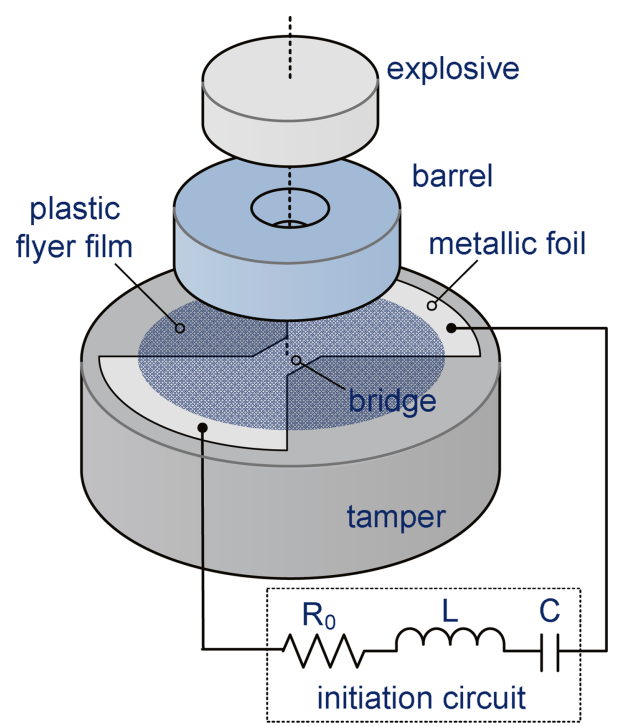

Fig. 1. Schematic diagram of exploding foil initiator with thin square metallic bridge.

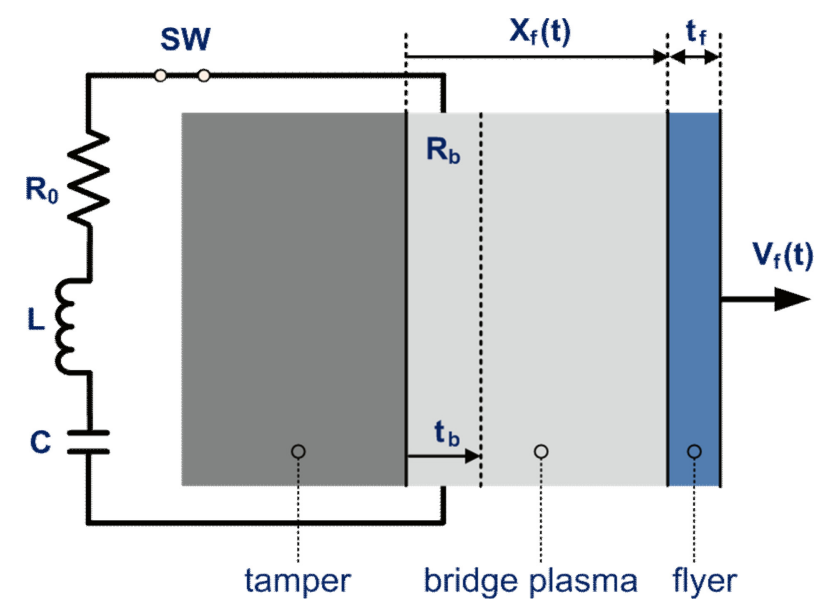

Fig. 2. Metallic plasma expansion of metallic bridge film during bridge burst.

a barrel over the thin film layers to guide the high speed flyer so that it impacts on an explosive charge.

In order to investigate the bridge burst characteristics, a theoretical model was established as follows. Assuming the spatial uniformity of the temperature, mass density, and pressure for the expanding bridge plasma inside the barrel hole as well as no frictional and thermal energy loss, the mass, momentum, and conservation principles for the bridge plasma and flyer lead to the following three equations in transient form, respectively [5]. 


$$
\begin{aligned}
& \rho(t) / \rho_{b}=t_{b} / X_{f}(t) \\
& p=\left(m_{f}+m_{b} / 2\right) d V_{f} / d t \\
& \frac{d e}{d t}=\frac{1}{\rho} \frac{J^{2}}{\sigma_{e, b}(T)}-\frac{p}{\rho} \frac{V_{f}}{X_{f}}-\left(\frac{1}{3}+\frac{m_{f}}{m_{b}}\right) V_{f} \frac{d V_{f}}{d t}
\end{aligned}
$$

In the above equations, $X_{f}$ and $V_{f}$ are the thickness of the expanding bridge material and flyer velocity $\left(V_{f}\right.$ $\left.=d X_{f} / d t\right)$, respectively, as illustrated in Fig. 2, while $\rho, T, p$, and $e$ are plasma density, temperature, pressure and internal energy, respectively. Also, $m_{b}$ and $m_{f}$ represent the bridge and flyer mass per unit area, as $\rho_{b}$ and $t_{b}$ are the bridge density and thickness, respectively, of the solid phase before the bridge burst. The treatment of joule heating term containing the bridge current density $(J)$ and electrical conductivity $\left(\sigma_{e, b}\right)$ in Eq. (3) requires coupling of the conservation principles with the following RLC circuit equation.

$$
L \frac{d I}{d t}+I\left(R_{0}+R_{b}\right)+\frac{1}{C}\left(\int_{0}^{t} I(\tau) d \tau+q_{0}\right)=0
$$

where $R_{0}$ and $R_{b}$ are the resistances of the initiation circuit and the metallic bridge, respectively. For the initial conditions for the above circuit equation, the initial electrical charge can be given as $q_{0}=V_{0} C$ with a charging voltage $V_{0}$ to capacitor $(C)$ in the device initiation circuit. The bridge resistance is modeled to be $R_{b}=1 / \sigma_{e, b} X_{f}$ by taking advantage of the simple bridge geometry. Note that dynamic bridge resistance depends strongly on the temperature of the bridge material, especially in the vapor and plasma states.

All those governing differential and algebraic equations of the RLC circuit and mass, momentum, and energy conservation principles in the EFI system can be integrated through time progress using any common initial value problem solver to simulate the temporal metallic bridge behaviors such as bridge current, resistance, and temperature. In this computational model, it is important to accurately provide the state equation and the electrical conductivity of the bridge plasma in Eqs. (3) and (4).
In this study, the plasma property calculations were performed by first finding the ionized metal species composition with Saha equations and then calculating the electrical conductivity of the metallic plasma under the assumption of local ionization equilibrium. Because highly dense metallic plasma is expected around the bridge burst, the present calculations employed a revised thermal plasma model on lowering of ionization energy, as recently proposed by Zaghloul [6] in order to account for possibly significant plasma non-ideality. The calculation of the electrical conductivity for metallic plasma vapor is based on the plasma property model of Zollweg and Liebermann [7], which accounts for electron collision with both types of heavy species such as ions and neutral atoms, if the dense metal vapor is partially ionized. The detailed procedures for these property computations of metallic plasma can be found in references $[8,9]$. When the bridge is in the condensed phase before the vaporization and bridge burst, the electrical conductivity is calculated using the algebraic property equations proposed by Burgess [4].

Since the thin film bridges are usually made of aluminum or copper, the present study considered these two types of metals for the bridge material and up to the third ionization level of monoatomic metal species are included here in the plasma property calculations $\left(\mathrm{Al}, \mathrm{Al}^{+}, \mathrm{Al}^{++}, \mathrm{Al}^{+++}\right.$or $\mathrm{Cu}, \mathrm{Cu}^{+}, \mathrm{Cu}^{++}$, $\left.\mathrm{Cu}^{+++}\right)$. Fig. 3 shows the mole fraction of neutral and

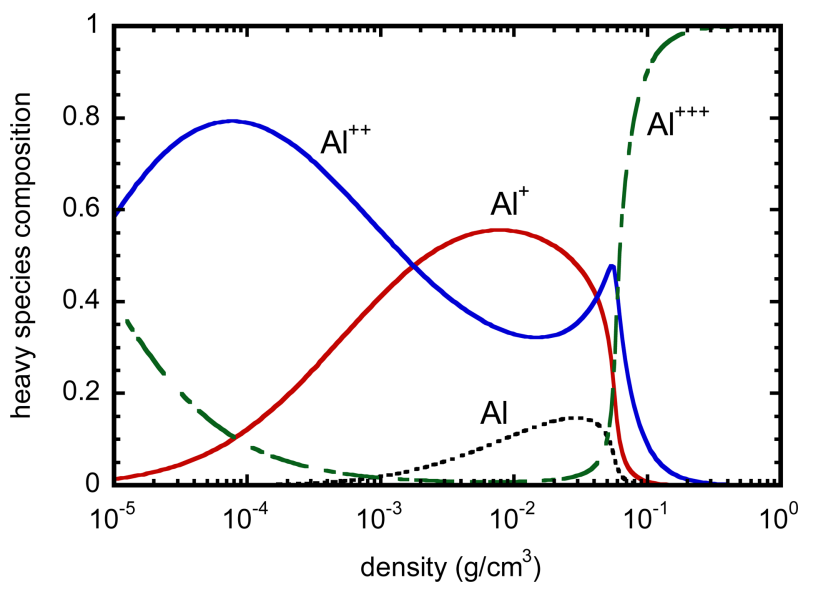

Fig. 3. Heavy species composition of aluminum plasma at temperatures of $31,600 \mathrm{~K}$. 


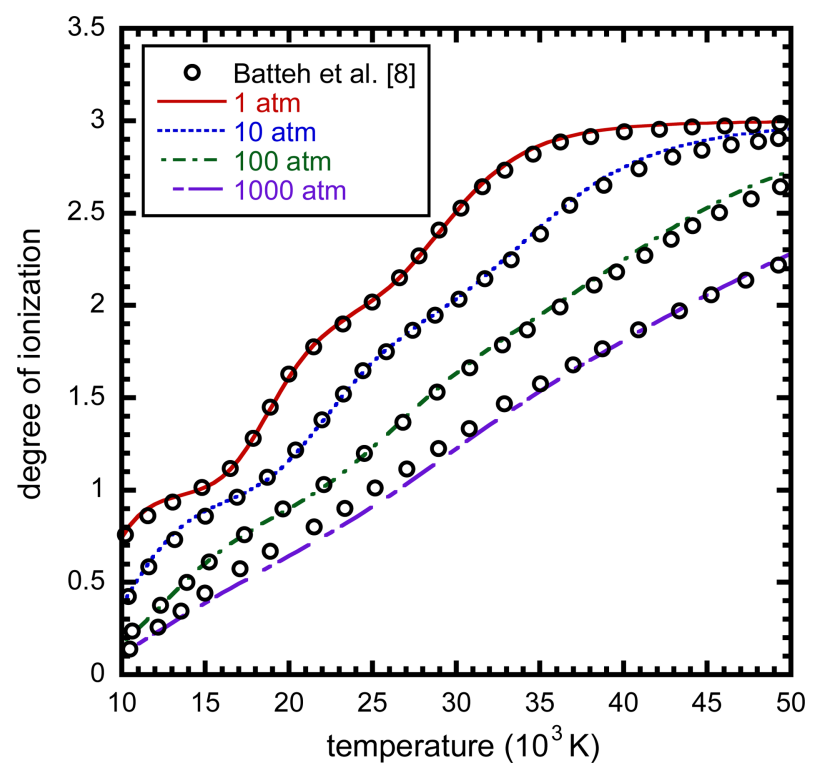

Fig. 4. Plasma degree of ionization for aluminum plasma at different levels of pressure.

ionized heavy species for aluminum plasma at temperature of $31,600 \mathrm{~K}$. In Fig. 4, the degree of ionization of the aluminum plasma can be seen as a function of temperature and pressure. It agrees well with the computed values reported by Batteh et al. [8] and this validates that the plasma property model provides the suitable state equation of the metallic plasma vapor. The calculated electrical conductivity of the aluminum plasma is presented with measured data $[10,11]$ for a wide range of plasma density at two temperatures of 14,000 and $31,600 \mathrm{~K}$ in Fig. 5. While the comparison with measurements is very favorable, the figures exhibit non-linearity for dense plasma, especially at lower temperature due to the aforementioned plasma non-ideality. Plasma properties for copper can be obtained using a similar method, and they were also compared and verified with corresponding measurement data, though these results are not shown here.

\section{RESULTS AND DISCUSSION}

The burst of a thin-film metallic bridge occurs when it is subjected to a high current density on the order of $0.1-1 \mathrm{TA} / \mathrm{m}^{2}$ [12] and it generates a violent
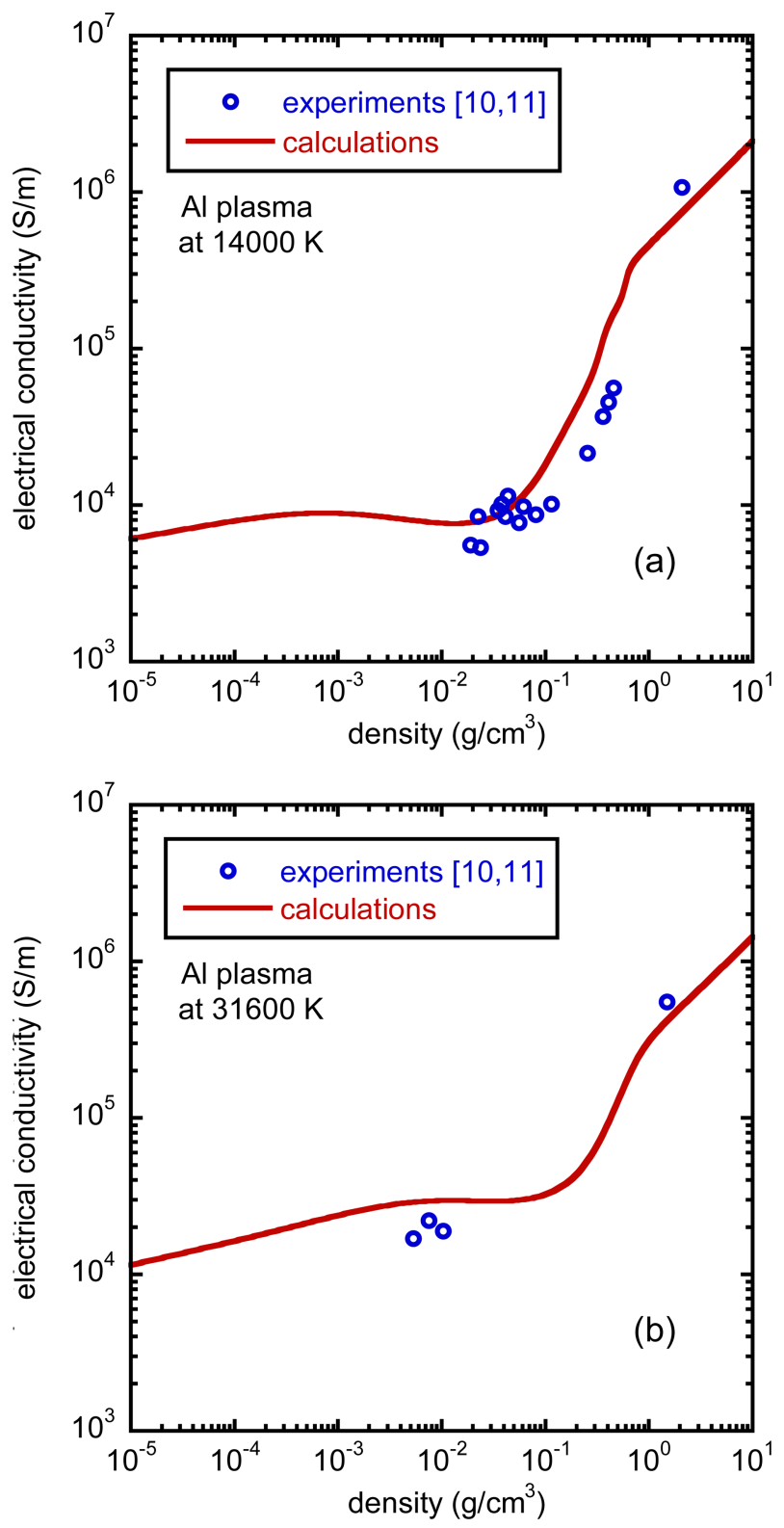

Fig. 5. Plasma electrical conductivity for aluminum plasma at temperatures of (a) $14000 \mathrm{~K}$ and (b) $31600 \mathrm{~K}$. Experimental data are from references $[10,11]$.

explosion of high temperature and high pressure plasma, which becomes the initiation sources of a shock wave (in EBF) or a high speed flyer creation out of plastic film (in EFI) for explosive charge detonation. The computational model of the bridge burst in an EFI system presented here was first applied to EFI firing tests and measurements by Logan et al. [13]. They investigated the bursts of aluminum and copper bridges, and tested nine cases 
to evaluate the effects of the bridge material, size, and thickness with varying charging voltages. The circuit parameters in the RLC type initiation circuit were $R_{0}=6 \mathrm{~m} \Omega, L=40 \mathrm{nH}$, and $C=56 \mu \mathrm{F}$, while the thickness of the polyimide flyer film was $250 \mu \mathrm{m}$.

For an aluminum bridge $51 \mu \mathrm{m}$ thick and $25.4 \times$ $25.4 \mathrm{~mm}^{2}$ in size, Fig. 6 shows the temporal traces of the bridge current and resistance at a charging voltage of $40 \mathrm{kV}$. The spikes in the predicted bridge resistance change clearly indicate the complex phenomena of the bridge burst, as the bridge undergoes phase change and vaporizes into an expanding plasma. The first and minor increase around $0.62 \mu \mathrm{s}$ is due to the bridge melting. The second and major increase in bridge resistance around $0.94 \mu \mathrm{s}$ is from explosive plasma expansion. The time of the bridge burst is determined by this major spike in bridge resistance. The predicted burst time is very close to the $1.00 \mu$ s reported in the measurements.

This effect can also be observed in the temporal change of the bridge current. Around the bridge burst time, an inflection point can be observed in both the

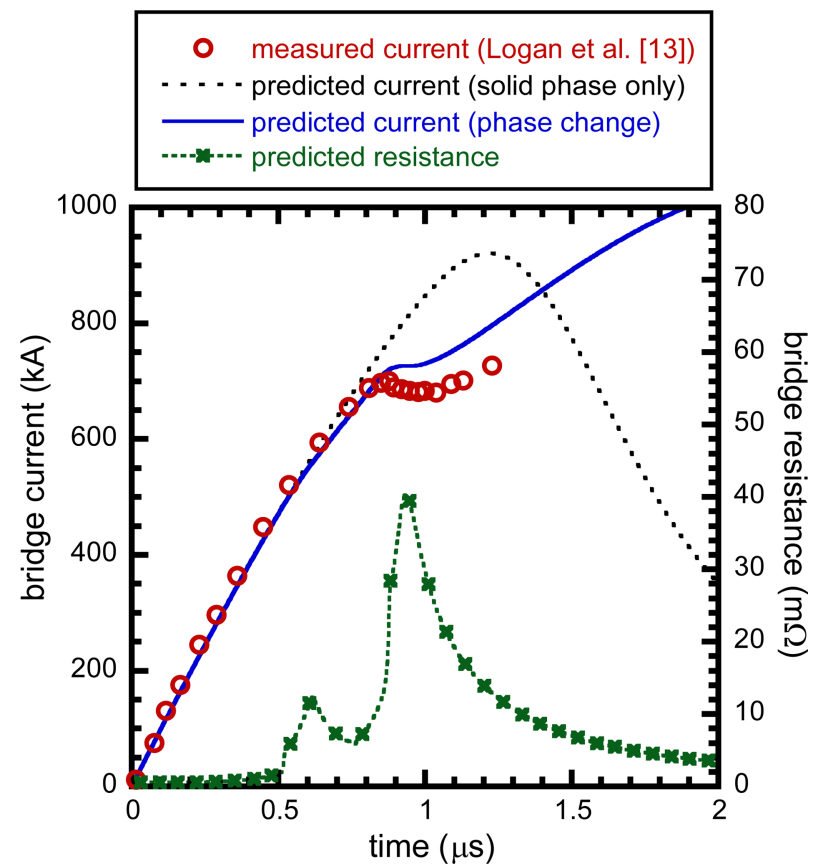

Fig. 6. Dynamic responses of aluminum bridge at charging voltage of $40 \mathrm{kV}$ in EFI detonation system. Bridge dimensions are $51 \mu \mathrm{m}$ thick and $25.4 \times 25.4 \mathrm{~mm}^{2}$ wide. measurement and prediction, as shown in Fig. 6. In fact, this is one of typical characteristics of a bridge burst, and it can be seen in many other measurements $[4,14]$. The bridge current density at bridge burst was predicted to be $0.56 \mathrm{TA} / \mathrm{m}^{2}$ which it is in excellent agreement with the measured value of $0.54 \mathrm{TA} / \mathrm{m}^{2}$.

The bridge burst tests conducted by Logan et al. [13] provided eight cases of measurements, based on changing the bridge thickness and size of the aluminum and copper thin film bridges at a charging
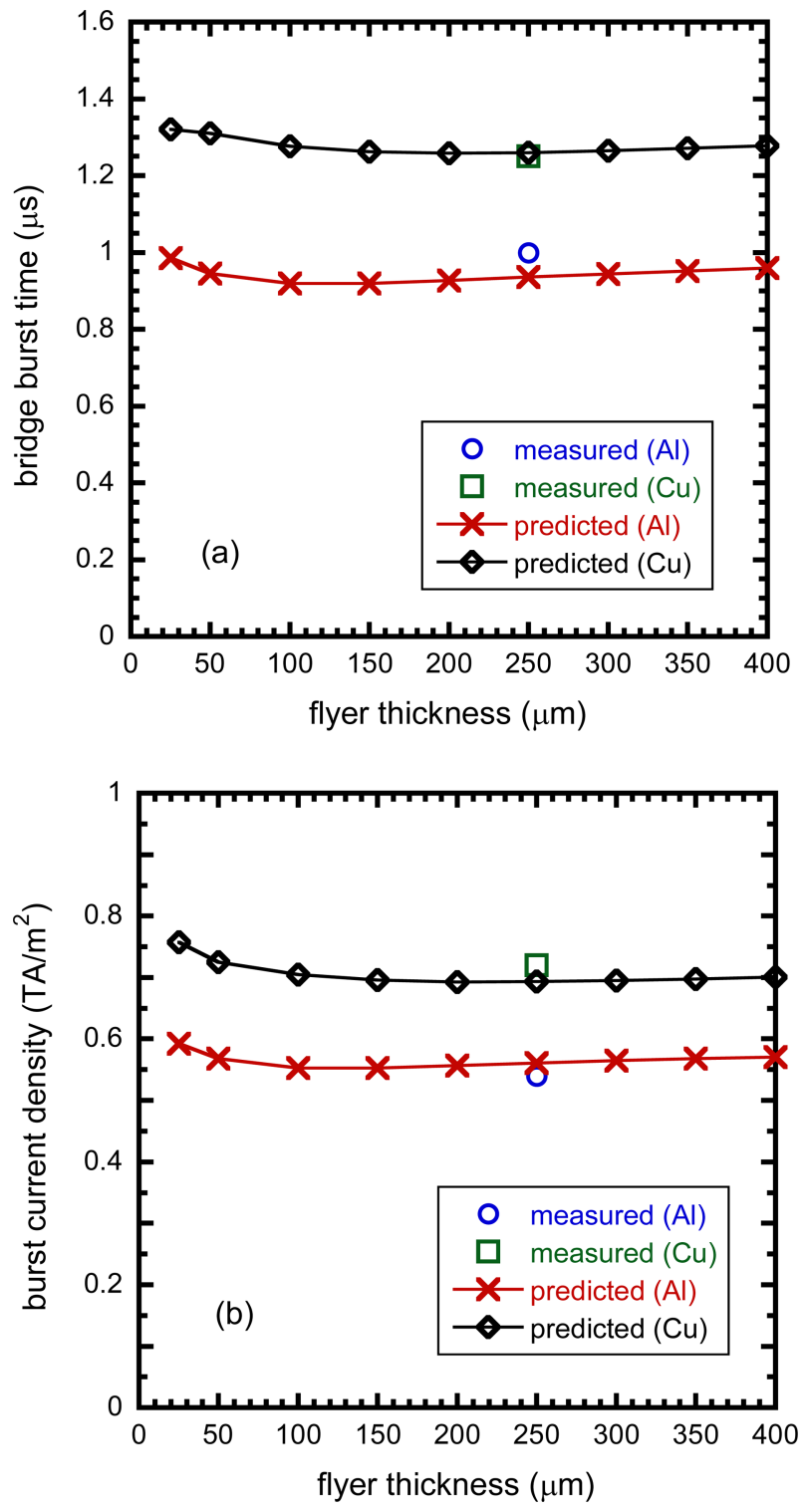

Fig. 7. Effects of flyer thickness on (a) burst time and (b) burst current density in aluminum and copper bridges at charging voltage of $40 \mathrm{kV}$. Measured data are from Logan et al. [13]. Bridge dimensions are $51 \mu \mathrm{m}$ thick and $25.4 \times 25.4 \mathrm{~mm}^{2}$ wide. 
voltage of $40 \mathrm{kV}$. They recorded the explosion properties of the metallic bridge, including the bridge burst time and burst current density for each case. The present theoretical model was validated using those measurement data.

Burst current density, which is based on the bridge current at the time of the bridge burst, is an important parameter for analyzing the effectiveness of the bridge explosion and is usually related to the flyer velocity through the Gurney equation [15]. Bridge burst time is also important for designing electrical initiators and this short duration delay can be controllable and useful for setting a precisely timed initiation of required actions at several different places in the system [14].

Figure 7 presents the effects of flyer thickness on bridge burst time and current density. It was found that neither the bridge burst time or burst density changed sensitively with flyer thickness, unless the flyer became very thin, under $30 \mu \mathrm{m}$. When both metals were compared to each other, the copper bridge gave a longer burst time and higher burst current density than the aluminum bridge. Figures 8 and 9 show the model validation using experimental data for different bridge size and thickness, and good agreement was found between prediction and measurement. As expected, employing thicker and larger square-shaped bridges resulted in delaying the bridge burst and lowering the burst current density. Here also, the copper bridge exhibited a significantly higher burst current density than the aluminum one, unless the bridge was unusually large or thick, in which case it was out of the practical size range. This gives copper advantages, making it a better bridge material.

In Fig. 10, changes in the bridge burst time and burst current density with charging voltage matched the measured data well for aluminum and copper bridges of $51 \mu \mathrm{m}$ thickness and $25.4 \times 25.4 \mathrm{~mm}^{2}$ width. Higher energy deposition with increased charging voltage leads to a shorter time delay of bridge burst and higher bridge burst current density. Other features which contribute to good explosion
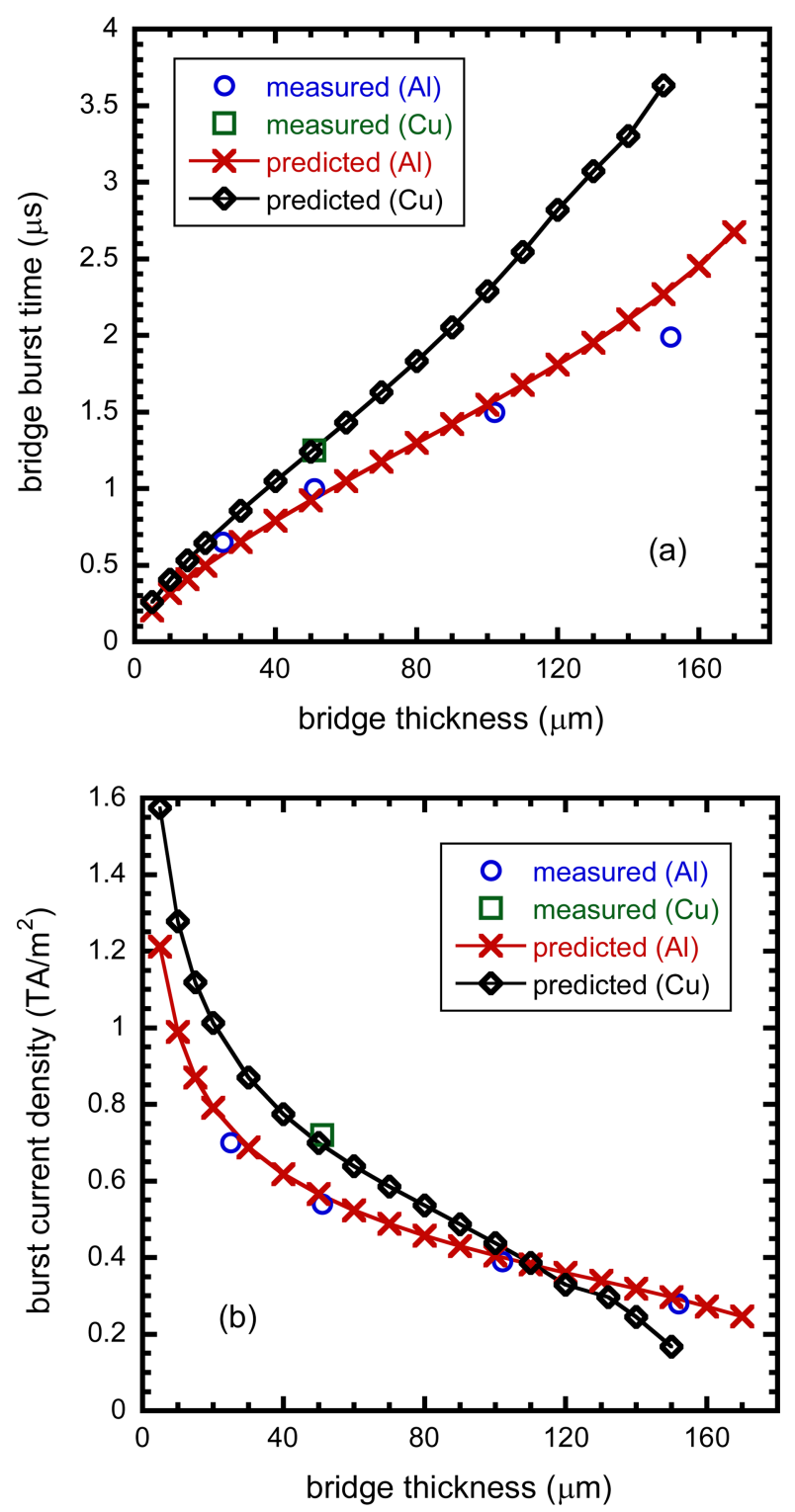

Fig. 8. Effects of bridge thickness on (a) burst time and (b) burst current density in aluminum and copper bridges at charging voltage of $40 \mathrm{kV}$. Measured data are from Logan et al. [13]. Bridge size is $25.4 \times 25.4 \mathrm{~mm}^{2}$ and flyer thickness is $250 \mu \mathrm{m}$.

properties in metallic bridges, especially for the detonation or ignition of high energy materials, would be the discrete nature of the bridge burst by electric input such as charging voltage, since device safety largely depends on its resistance to unintended static electricity or electromagnetic interference. For given bridge dimensions and material, there exists a lowest charging voltage for creating a bridge burst. It can be found by checking the significant spike in bridge 

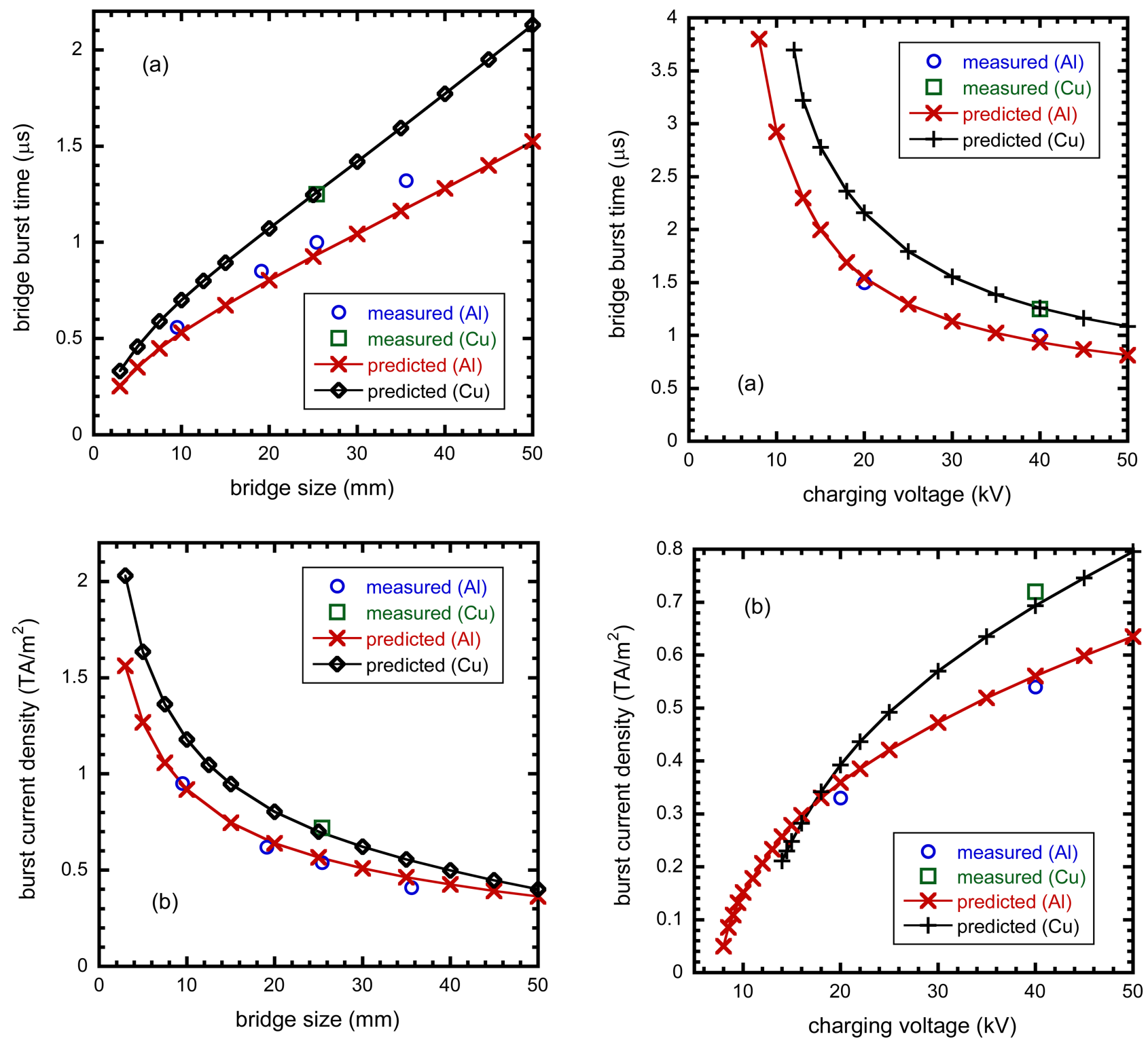

Fig. 9. Effects of bridge size on (a) burst time and (b) burst current density in aluminum and copper bridges at charging voltage of 40 $\mathrm{kV}$. Measured data are from Logan et al. [13]. Bridge and flyer thicknesses are $51 \mu \mathrm{m}$ and $250 \mu \mathrm{m}$, respectively.

resistance in the measurements or predictions. While the aluminum bridge can produce a bridge burst with a charging voltage as low as $8 \mathrm{kV}$, the threshold charging voltage for the copper bridge is approximately $14 \mathrm{kV}$. This moderately high threshold of working voltage is one of the important parameters to be considered when designing the electrical initiation system. It permits a high margin of safety without auxiliary mechanical barriers, thus, providing

Fig. 10. Change of (a) burst time and (b) burst current density with charging voltage in aluminum and copper bridges. Measured data are from Logan et al. [13]. Bridge dimensions are $51 \mu \mathrm{m}$ thick and $25.4 \times 25.4 \mathrm{~mm}^{2}$ wide.

the combined benefits of higher reliability and lower manufacturing cost.

With continuing advances in MEMS manufacturing, the electrical initiators are also being miniaturized, and the bridge dimensions are now below thicknesses of $10 \mu \mathrm{m}$ and sizes of one millimeter $[2,15,16]$. So far, validation of the prediction model has only been performed with rather large sized metallic bridges and, therefore, model validity should also be tested with 


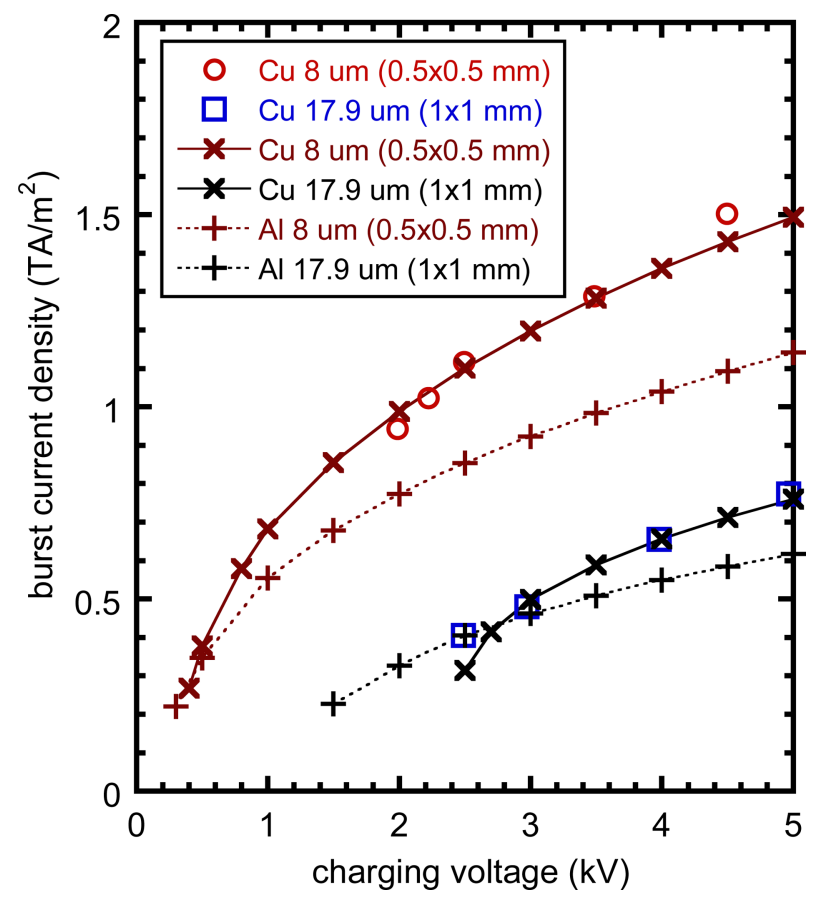

Fig. 11. Change of burst current density with charging voltage in small sized aluminum and copper bridges. Measured data are from Hasman et al. [17] with copper bridge. Filled symbols represent measurements and lines are from the predictions.

small sized bridges. Hasman et al. [17] made a series of EFI firing tests and provided the bridge burst current for small sized copper bridges. The circuit parameters in their initiation circuit were given as $R_{0}$ $=76 \mathrm{~m} \Omega, L=200 \mathrm{nH}$, and $C=3 \mu \mathrm{F}$.

Fig. 11 shows the bridge burst current density with changing voltage up to $5 \mathrm{kV}$ for two sizes of bridges: $0.5 \times 0.5 \mathrm{~mm}^{2}$ and $8 \mu \mathrm{m}$ thick; $1 \times 1 \mathrm{~mm}$ and $17.9 \mu \mathrm{m}$ thick. The flyer film is polyimide and its thickness is $250 \mu \mathrm{m}$. The effects of the charging voltage and different bridge material remain similar to those of larger bridge sizes, and the agreement between prediction and measurement is also very favorable. Here, the charging voltage threshold was found to be higher in the copper bridges than the aluminum ones. The bridge burst current density of the copper bridge was also significantly higher than that of the aluminum bridge. Thus, the copper bridge is expected to be more suitable as a bridge film material due to better explosion properties of bridge burst in the aspect of burst current density and threshold voltage, as these properties indicate reliable explosive initiation and high safety against unwanted electrical interference.

\section{CONCLUSIONS}

In this paper, the bridge bursts of thin film aluminum and copper bridges were numerically modeled and investigated in order to understand and compare the explosion properties of the aluminum and copper bridge materials. The burst prediction model was validated using measured data from electrical initiation system tests, and the model was found to be very suitable for predicting important explosion parameters, such as bridge burst time and burst current density, even for thin film bridge under one millimeter size and $10 \mu \mathrm{m}$ thickness. The comparisons showed that the copper bridges had a longer burst delay but significantly higher bridge burst density, as well as higher charging voltage threshold for bridge burst. These features make the copper bridges more suitable than the aluminum bridges, ensuring better initiation performance and safety features in electrical initiation devices.

\section{ACKNOWLEDGEMENTS}

This research has been supported by Defense Acquisition Program Administration (DAPA) and Agency for Defense Development (ADD) in Korea.

\section{REFERECES}

1. J. E. Osher, G. Barnes, H. H. Chau, R. S. Lee, C. Lee, R. Speer, and R. C. Weingart, IEEE Trans. Plasma Sci. 17, 392 (1989).

2. Q.-C. Chen, Q.-B., Fu, L. Chen, and Z.-F. Han, Propell. Explos. Pyrot. 35, 558 (2013).

3. J. L. Zirnheld, S. Olabisi, K. Burke, T. M. Disanto, H. L. Moore, and H. Singh, IEEE Trans. Plasma Sci. 37, 2378 (2009).

4. B.-Q. Luo, C.-W. Sun, J.-H. Zhao, and J. He, IEEE Trans. Plasma Sci. 41, 49 (2013).

5. K. Kim, K.-H. Kim, and S.-G. Jang, Proceedings of the 2016 Asia-Pacific International Symposium on Aerospace 
Technology, Toyama, Japan (2016).

6. M. R. Zaghloul, Phys. Plasmas 15, 042705 (2008).

7. R. J. Zollweg and R. W. Liebermann, J. Appl. Phys. 62 , 3621 (1987).

8. J. Batteh, J. Powell, D. Sink, and L. Thornhill, IEEE Trans. Magn. 31, 388 (1995).

9. K. Kim, IEEE Trans. Plasma Sci. 31, 729 (2003).

10. A. W. DeSilva and J. D. Katsouros, Phys. Rev. E 57, 5945 (1998).

11. J. F. Benage, W. R. Shanahan, and M. S. Murillo, Phys. Rev. Lett. 83, 2953 (1999).

12. T. M. Disanto, S. Olabisi, D. P. Muffoletto, K. Burke, H. L. Moore, H. Singh, and J. L. Zirnheld, IEEE Trans. Plasma
Sci. 39, 603 (2011).

13. J. D. Logan, R. S. Lee, R. C. Weingart, and K. S. Yee, J. Appl. Phys. 48, 621 (1977).

14. L. L. Altgilbers, J. Baird, B. L. Freeman, C. S. Lynch, and S. I. Shkuratov, Explosive Pulsed Power, pp. 74-80, Imperial College Press, London (2010).

15. L. Nappert, DREV-R-9502, Defense Research Establishment Valcartier (1996).

16. Q. Zeng, B. Li, M. Li, and X. Wu, Propell. Explos. Pyrot. 41, 864 (2016).

17. E. Hasman, M. Gvishi, and Y. Carmel, Propell. Explos. Pyrot. 11, 144 (1986). 